\title{
Systèmes MIMO et codage spatio-temporel
}

\author{
Didier Le Ruyet ${ }^{\dagger}$ et Berna Özbek ${ }^{\dagger}$ \\ $\dagger$ CNAM, 292 rue Saint Martin,75141 Paris Cedex 3, France \\ téléphone: 0140272590, télécopie: 0140272779, email: leruyet@cnam.fr
}

\begin{abstract}
Cet article présente une synthèse des systèmes de transmission sans fil à plusieurs antennes à l'émission et à la réception. Après avoir introduit les capacités théoriques des systèmes multi-antennes, nous présentons différentes familles de codes spatio-temporels et d'algorithmes de décodage associés. Ces codes permettent d'exploiter les gains de multiplexage et de diversité des systèmes multi-antennes. Nous montrerons que lorsque les codes spatio-temporel sont associés à des codes externes, il est alors possible de s'approcher de la capacité théorique des systèmes multi-antennes. Nous considérerons également le cas des canaux sélectifs en fréquence où les codes spatio-temporels sont alors combinés avec la technique OFDM. Finalement nous présenterons quelques unes des applications envisagées.
\end{abstract}

keywords : système de radiocommunication, canal à évanouissement, antennes multiples, codage de canal, capacité

\section{INTRODUCTION}

Les systèmes multi-antennes à l'émission et à la réception (Multi input multi output MIMO en anglais) permettent théoriquement d'accroître la capacité des liens de communications sans fil par rapport aux systèmes composés d'une seule antenne à l'émission et à la réception (Single input Single output SISO en anglais). En faisant l'hypothèse que les trajets entre chaque antenne d'émission et de reception sont indépendants, Foshini [6] et Telatar [17] ont démontré que la capacité théorique du canal MIMO avec $N_{t}$ antennes à l'émission et $N_{r}$ antennes à la réception croit linéairement avec $\min \left(N_{t}, N_{r}\right)$. Les systèmes MIMO sont l'un des principaux axes de développement pour augmenter les débits des communications sans fil et bien que les premiers travaux publiés sur ce sujet ne datent que de quelques années, nous assistons à un très rapide développement de cette technologie avec des applications déjà envisagées dans les réseaux locaux sans fil et les réseaux de communication de 3ème génération. Les systèmes MIMO sont par exemple proposés pour le futur standard de réseau local sans fil IEEE 802.11n où l'objectif est d'atteindre des débits de 100 mégabits par seconde pour les applications vidéo.

Les systèmes MIMO présentent deux avantages majeurs par rapport aux systèmes SISO : 1) grâce à l'apport de la diversité spatiale ils permettent d'améliorer la qualité du lien en s'affranchissant des évanouissements des canaux 2) par multiplexage spatial, ils permettent d'augmenter le débit d'information sans augmenter la bande passante ou la puissance transmise.

Le principe de base des systèmes MIMO consiste donc à combiner les signaux judicieusement tant à l'émission qu'à la réception pour exploiter la diversité spatiale et donc réduire les

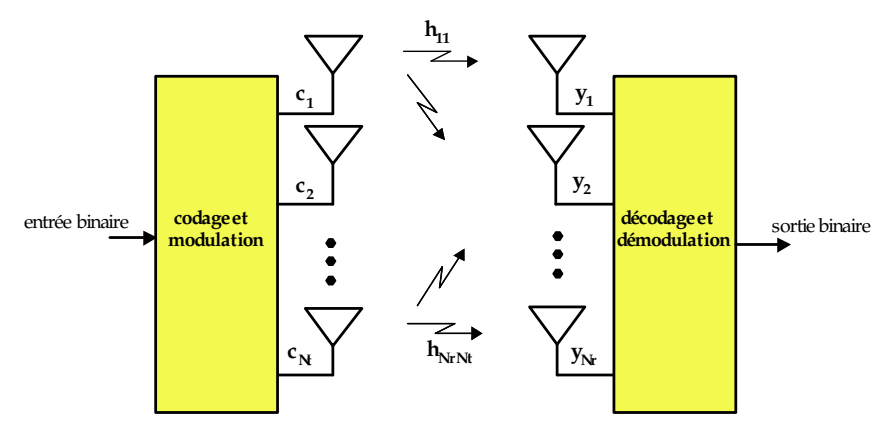

Fig. 1. système de transmission sans fil MIMO

effets des évanouissements ou pour augmenter le débit de transmission.

Dans cet article, après avoir introduit les systèmes MIMO et leurs capacités nous nous intéresserons aux procédés de codage et de décodage permettant d'atteindre les limites théoriques. Finalement nous présenterons les avancées récentes et les travaux de standardisation en cours de développement.

\section{CAPACitÉ ET GAIN DES SYSTÈMES MIMO}

Soit un système MIMO composé de $N_{t}$ antennes à l'émission et de $N_{r}$ antennes à la réception comme illustré sur la figure 1.

Le signal reçu $y_{j}$ à chaque instant sur la $j$-ième antenne de réception est la somme des symboles bruités issus des $N_{t}$ signaux transmis :

$$
y_{j}=\sum_{i=1}^{N_{t}} h_{j i} c_{i}+n_{j}
$$

où $h_{j i}$ est le gain du canal non sélectif en fréquence entre l'antenne d'émission $i$ et l'antenne de réception $j$ et $n_{j}$ est le bruit additif qui est modélisé par des échantillons indépendants et suivant une loi gaussienne centrée de variance $N_{0} / 2$ par dimension réelle. Le rapport signal à bruit $(\mathrm{RSB})$ par antenne de réception est défini par $R S B=E_{s} / N_{0}$.

Soit la matrice du canal MIMO $\mathbf{H}$ de dimension $N_{r} \times N_{t}$ suivante :

$$
\mathbf{H}=\left[\begin{array}{ccc}
h_{11} & \ldots & h_{1 N_{t}} \\
\vdots & \ddots & \vdots \\
h_{N_{r} 1} & \ldots & h_{N_{r} N_{t}}
\end{array}\right]
$$

L'équation (1) peut s'écrire sous une forme matricielle comme suit:

$$
\mathbf{y}=\mathbf{H c}+\mathbf{n}
$$

où $\mathbf{y}$ et $\mathbf{n}$ sont respectivement les vecteurs de réception et de bruit de dimension $N_{r} \times 1$. 


\section{A. Capacité d'un canal MIMO}

Considérons un système MIMO avec $N_{t}$ antennes d'émission et $N_{r}$ antennes de réception. On suppose que le récepteur connaît parfaitement le canal alors que l'émetteur ne dispose pas de la connaissance du canal.

Le canal MIMO défini par la matrice $\mathbf{H}$ peut être décomposé en plusieurs canaux (SISO) parallèles en utilisant la décomposition en valeurs propres (SVD) comme suit

$$
\mathbf{H}=\mathbf{U} \Sigma \mathbf{V}^{H}
$$

où $\mathbf{U}$ et $\mathbf{V}$ sont des matrices unitaires et $\Sigma$ est la matrice diagonale $\Sigma=\operatorname{diag}\left(\sqrt{\lambda_{1}}, \sqrt{\lambda_{2}}, \ldots, \sqrt{\lambda_{r}}, 0, \ldots, 0\right)$ où $\lambda_{i}(i=1, \ldots, r)$ sont les valeurs propres non nulles de $\mathbf{H}^{H} \mathbf{H}$ (en considérant que $N_{t} \leq N_{r}$ ). Le nombre de valeurs propres $r$ est le rang de la matrice de canal $\mathbf{H}$ et est égal à $\min \left(N_{t}, N_{r}\right)$.

En appliquant un pré-traitement aux symboles transmis $(\mathbf{V c})$ du coté de l'émetteur et un post-traitement à la réception $\left(\mathbf{U}^{H} \mathbf{y}\right)$ on obtient la relation

$$
\begin{gathered}
\mathbf{U}^{H} \mathbf{y}=\mathbf{U}^{H}\left(\mathbf{U} \Sigma \mathbf{V}^{H}\right) \mathbf{V c}+\mathbf{U}^{H} \mathbf{n} \\
\tilde{\mathbf{y}}=\Sigma \tilde{\mathbf{c}}+\tilde{\mathbf{n}}
\end{gathered}
$$

où $\tilde{\mathbf{n}}$ est encore gaussien avec la même variance que $\mathbf{n}$.

L'équation (5) représente un système équivalent avec $r$ canaux SISO parallèle dont la puissance du signal est donnée par leurs valeurs propres. Ainsi, la capacité instantanée peut être écrite ainsi :

$$
C(\rho)=\sum_{i=1}^{r} \log _{2}\left(1+\frac{\rho}{N_{t}} \lambda_{i}\right)
$$

Cette équation peut également être décrite comme suit [17] [6]

$$
C\left(\rho, N_{t}, N_{r}\right)=\log _{2} \operatorname{det}\left(\mathbf{I}_{N_{r}}+\frac{\rho}{N_{t}} \mathbf{H H}^{H}\right)
$$

où $\rho=E_{s} / N_{0}$ est le rapport signal à bruit

Comme la capacité instantanée donnée en (6) et (7) est une variable aléatoire, en pratique, on utilise deux formes plus pratiques pour décrire la capacité : la capacité moyenne et la capacité de coupure.

La capacité moyenne ou ergodique s'obtient en calculant l'espérance sur toutes les réalisations possibles du canal MIMO.

$$
C\left(\rho, N_{t}, N_{r}\right)=E\left\{\log _{2} \operatorname{det}\left(\mathbf{I}_{N_{r}}+\frac{\rho}{N_{t}} \mathbf{H H}^{H}\right)\right\}
$$

Si la durée du bloc d'information est limitée devant le temps de cohérence du canal, on utilise la capacité de coupure $q \%$

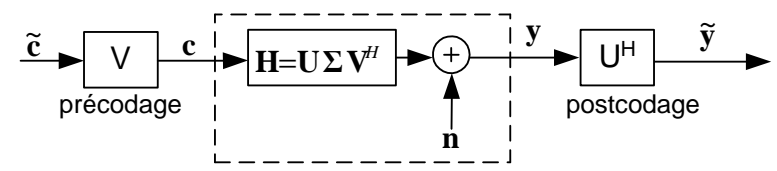

Fig. 2. décomposition SVD du canal MIMO

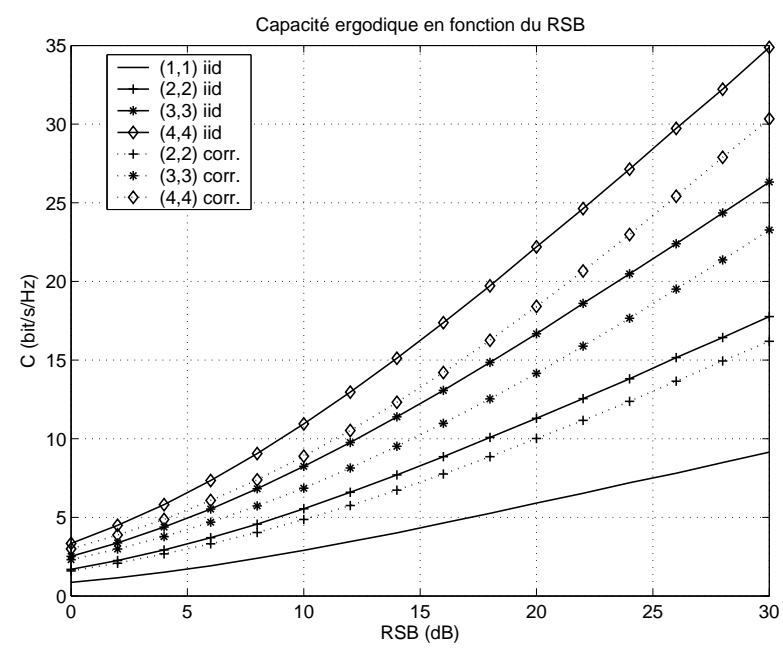

Fig. 3. Capacité ergodique pour différentes configurations

$C_{\text {out }, q}$. Elle est définie comme le débit d'information garanti pour $(100-q) \%$ des réalisations du canal, i.e, $P(C \leq$ $\left.C_{\text {out }, q}\right)=q \%$.

Comme la capacité instantanée, la capacité ergodique et la capacité de coupure sont exprimées en bit par seconde et par Hertz (bit/s/Hz).

Sur la figure (3) nous présentons la capacité ergodique pour différentes configurations en fonction du rapport RSB. Les canaux de transmission utilisés pour évaluer ces capacités sont des canaux non sélectifs en fréquence, indépendants et identiquement distribués (i.i. d) suivant une loi de Rayleigh et varient pour chaque bloc. On peut vérifier que la capacité croît en fonction de $\min \left(N_{t}, N_{r}\right)$ (chaque accroissement de $3 \mathrm{~dB}$ implique $\min \left(N_{t}, N_{r}\right) \mathrm{bit} / \mathrm{s} / \mathrm{Hz}$ en plus) . Nous présentons également les capacités ergodiques obtenues lorsque les canaux de transmission sont corrélés (lien montant, à l'émission : distance entre antenne $=0.5 \lambda$, angle de départ $=20^{\circ}$, à la réception distance entre antenne $=4.0 \lambda$, angle d'arrivée $=50^{\circ}$, angle de dispersion azimutal $=5^{\circ}$ ). On peut observer que la corrélation réduit très sensiblement la capacité des canaux MIMO). Dans l'étude de la capacité des canaux de transmission, le choix du modèle de propagation est important. Cependant, les mesures expérimentales ont montré une bonne adéquation avec les évaluations théoriques de la capacité.

Finalement, il est important de signaler que lorsque le canal MIMO est connu à l'émission, la capacité du canal MIMO est sensiblement augmentée ( 10 à $30 \%$ de gain). L'exploitation de ce gain implique cependant d'utiliser un algorithme de remplissage (water filling).

\section{B. Gain des systèmes MIMO}

1) Gain de diversité spatiale: Dans un canal de transmission sans fil, la puissance reçue varie dans le temps, en fréquence et dans l'espace. La diversité est alors utilisée pour combattre ces évanouissements. L'idée principale consiste à utiliser à la réception plusieurs répliques du signal. Plus le nombre de répliques augmente, plus la probabilité que toutes les répliques subissent simultanément un évanouissement diminue. 
Classiquement, dans les systèmes SISO les diversités temporelle et fréquentielle sont exploitées par le codage de canal associé à une fonction d'entrelacement. Avec les systèmes MIMO, on dispose d'une nouvelle forme de diversité : la diversité spatiale. L'ordre de diversité est fonction du nombre d'antennes à l'émission et à la réception. En pratique, pour mettre à profit cette diversité, il est nécessaire d'utiliser un code spatio-temporel.

La diversité joue sur la pente de la courbe $\mathrm{TEB}=\mathrm{f}(\mathrm{RSB})$ $\left(T E B \propto R S B^{-r}\right.$ pour une diversité d'ordre r)

2) Gain de multiplexage: Lorsque les évanouissements des différents canaux sont indépendants, on peut montrer que la matrice de canal est de rang plein avec une grande probabilité. Il est alors possible de voir le canal MIMO comme un ensemble de canaux SISO en parallèle. En transmettant des flux d'information dans chacun de ces canaux, il est possible d'augmenter le débit d'information. Il en résulte un gain dit de multiplexage. Ce gain est limité par $\min \left(N_{t}, N_{r}\right)$.

Dans le reste de cet article, nous allons présenter des systèmes MIMO qui exploitent les gains de diversité et de multiplexage.

Il existe deux approches pour exploiter le potentiel des canaux MIMO : le multiplexage spatial et le codage spatiotemporel. Dans le multiplexage spatial, des flux de données indépendants sont transmis sur les différentes antennes d'émission maximisant ainsi le débit transmis. A l'opposé, les codes spatio-temporel offrent à la fois de la diversité et du gain de codage tout en améliorant l'efficacité spectrale.

\section{CRITÈRES POUR LA CONSTRUCTION DES CODES SPATIO-TEMPOREL}

Dans ce paragraphe, nous introduirons les critères utilisés pour construire de bons codes spatio-temporels.

Soit un système MIMO composé de $N_{t}$ antennes à l'émission et de $N_{r}$ antennes à la réception.

On supposera que les coefficients du canal MIMO restent constants pendant $T$ intervalles de temps élémentaires (modèle de canaux à évanouissement par bloc).

A l'émission, les symboles d'information $s_{i}$ appartenant à l'alphabet $\Lambda_{s}$ sont groupés en bloc $\mathbf{s}=\left[s_{1}, s_{2}, \ldots, s_{Q}\right]^{T}$ de dimension $Q \times 1$. Ce bloc est encodé par l'encodeur qui associe s à la matrice code suivante de dimension $N_{t} \times T$ :

$$
\mathbf{C}=\left[\begin{array}{ccc}
c_{11} & \cdots & c_{1 T} \\
\vdots & \ddots & \vdots \\
c_{N_{t} 1} & \cdots & c_{N_{t} T}
\end{array}\right]
$$

où le symbole codé $c_{i j}$ appartient à l'alphabet $\Lambda_{c}$. le rendement du code MIMO code est égal à $R_{M I M O}=Q / T$.

En considérant que le canal est non sélectif en fréquence, à partir de la relation (1) on peut écrire la relation matricielle suivante:

$$
\mathbf{Y}=\mathbf{H C}+\mathbf{N}
$$

où $\mathbf{Y}$ et $\mathbf{N}$ sont respectivement les matrices de réception et de bruit de dimension $N_{r} \times T$.

Nous définissons la probabilité d'erreurs par paire pour une réalisation du canal $\mathbf{H} P\left\{\mathbf{C} \rightarrow \mathbf{C}^{\prime} \mid \mathbf{H}\right\}$ comme la probabilité que le récepteur décode le bloc $\mathbf{C}^{\prime}$ alors que le bloc $\mathbf{C}$ a été transmis.

Soit la matrice de différence

$$
\mathbf{B}=\left[\begin{array}{ccc}
c_{11}-c_{11}^{\prime} & \ldots & c_{1 T}-c_{1 T}^{\prime} \\
c_{21}-c_{21}^{\prime} & \ldots & c_{2 T}-c_{2 T}^{\prime} \\
\cdot & \cdot & \cdot \\
c_{N_{t} 1}-c_{N_{t} 2}^{\prime} & \ldots & c_{N_{t} 2}-c_{N_{t} 2}^{\prime}
\end{array}\right]
$$

Comme la matrice $\mathbf{A}=\mathbf{B B}^{H}$ est hermitique, il existe une matrice unitaire $\mathbf{V}$ et une matrice réelle diagonale $\mathbf{D}$ tel que $\mathbf{V A V}^{H}=\mathbf{D}$. Les éléments de la diagonale de $\mathbf{D}$ sont les valeurs propres de $\mathbf{A}$, i.e. $\lambda_{i} ; i=1,2, . ., N_{t}$.

On peut montrer que la probabilité $P\left(\mathbf{C} \rightarrow \mathbf{C}^{\prime} \mid \mathbf{H}\right)$ peut être bornée supérieurement comme suit :

$$
\begin{array}{r}
P\left(\mathbf{C} \rightarrow \mathbf{C}^{\prime} \mid \mathbf{H}\right) \leq \exp \left(-\frac{E_{s}}{4 N_{0}} d^{2}\left(\mathbf{C}, \mathbf{C}^{\prime}\right)\right) \\
d^{2}\left(c, c^{\prime}\right)=\sum_{j=1}^{N_{r}} \mathbf{h}_{j} \mathbf{B B}^{H} \mathbf{h}_{j}^{H}=\sum_{j=1}^{N_{r}} \sum_{i=1}^{N_{t}} \lambda_{j}\left|\beta_{i j}\right|^{2}
\end{array}
$$

où $\mathbf{h}_{j}=\left[\begin{array}{llll}h_{1 j} & h_{2 j} & \ldots & h_{N_{t} j}\end{array}\right]$ est la transposition de la j-ième colonne de $\mathbf{H}$.

Après moyennage sur l'ensemble des réalisations des canaux à évanouissement, on montre que la probabilité par paire $P\left\{\mathbf{C} \rightarrow \mathbf{C}^{\prime}\right\}$ peut être bornée supérieurement par

$$
P\left(\mathbf{C} \rightarrow \mathbf{C}^{\prime}\right) \leq\left(\frac{E_{s}}{4 N_{0}}\right)^{-r_{d} N_{r}}\left(\prod_{k=1}^{r_{d}} \lambda_{k}\right)^{-N_{r}}
$$

où $r_{d}$ est le rang de la matrice $\mathbf{A}$ et $\lambda_{k}$ correspond aux valeurs propres non nulles de $\mathbf{B}$.

L'objectif est alors de construire un codeur optimal c'est-àdire minimisant la probabilité d'erreurs par paire $P\left\{\mathbf{C} \rightarrow \mathbf{C}^{\prime}\right\}$ pour toutes les paires possibles [16].

De cette expression on dérive deux critères de construction ; le critère de rang et de déterminant qui détermine respectivement le gain de diversité et de codage.

Critère du rang: Afin d'obtenir le degré maximum de diversité $N_{t} N_{r}$, la matrice de différence $\mathbf{B}$ doit avoir un rang plein pour toutes les paires distinctes de mot de code. Si le rang minimum est égal à $r_{d}$, le gain de diversité sera égal à $r_{d} N_{r}$.

Critère du déterminant: le terme $\prod_{k=1}^{r_{d}} \lambda_{k}$ représente le gain de codage. Celui-ci doit être maximisé pour l'ensemble de toutes les paires de matrices codes.

\section{MultipleXage spatial}

Le multiplexage spatial permet de maximiser le débit d'information sur un canal MIMO. Cette technique a été introduite sous le nom de BLAST (Bell Labs Layered Space-Time) dans [7]. Bien qu'il existe différentes versions, la version la plus populaire est la structure V-BLAST (vertical BLAST) où chaque couche est associée à une seule antenne de transmission. 


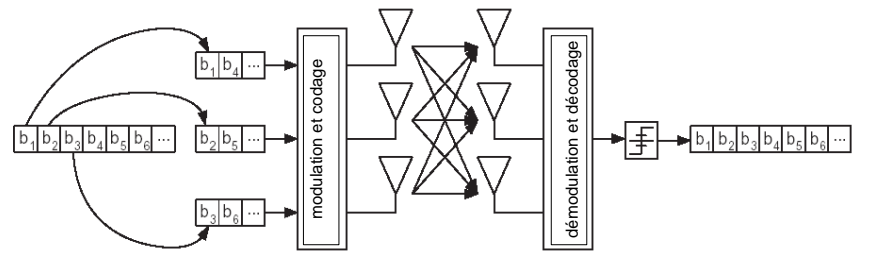

Fig. 4. synoptique de la technique V-BLAST

A titre d'exemple, nous allons considérer le cas où $N_{t}=$ $N_{r}=2, Q=2, T=1$ et donc $R_{M I M O}=2$, la matrice de code est la suivante,

$$
\mathbf{C}_{V B L A S T, 2}=\left[\begin{array}{l}
s_{1} \\
s_{2}
\end{array}\right]
$$

Le signal reçu s'écrit alors :

$$
\left[\begin{array}{l}
y_{11} \\
y_{21}
\end{array}\right]=\mathbf{H}\left[\begin{array}{l}
s_{1} \\
s_{2}
\end{array}\right]+\left[\begin{array}{l}
n_{11} \\
n_{21}
\end{array}\right]
$$

Pour pouvoir effectuer le décodage, le système doit être bien posé c'est-à-dire que $N_{r} \geq N_{t}$ et que la matrice du canal MIMO doit être de rang plein avec une très grande probabilité.

Outre les algorithmes de décodage linéaires comme le forçage à zéro $(\mathrm{ZF})$ ou utilisant le critère de la minimisation de l'erreur quadratique moyenne (MMSE), il est possible d'effectuer un décodage par soustraction successive des interférences (SIC). Cette technique est une généralisation de l'égaliseur à boucle de retour de décision. Le principe consiste après avoir mis le système sous une forme matricielle triangulaire supérieure, à estimer une première donnée puis à soustraire sa contribution avant d'estimer la suivante. Après avoir effectué une décomposition $\mathrm{QR}$ de la matrice du canal $\mathbf{H}=\mathbf{Q R}$ où $\mathbf{Q}$ est une matrice unitaire et $\mathbf{R}$ est une matrice triangulaire supérieure, on calcule les deux matrices suivantes :

$$
\left\{\begin{array}{l}
\mathbf{G}=\operatorname{diag}^{-1}(\mathbf{R}) \mathbf{Q}^{H} \\
\mathbf{L}=\operatorname{diag}^{-1}(\mathbf{R}) \mathbf{R}-\mathbf{I}_{N_{r}}
\end{array}\right.
$$

En multipliant le vecteur reçu par $\mathbf{G}$, on obtient la relation suivante :

$$
\tilde{\mathbf{y}}=\mathbf{G y}=\operatorname{diag}^{-1}(\mathbf{R}) \mathbf{R} \mathbf{s}+\mathbf{G n}
$$

Ainsi, il est possible d'estimer successivement les symboles $\mathbf{s}_{N_{t}}, \mathbf{s}_{N_{t}-1}, \ldots, \mathbf{s}_{1}$ :

$$
\left\{\begin{array}{l}
\hat{\mathbf{s}}_{N_{t}}=\text { décision }\left((\tilde{\mathbf{y}})_{N_{t}}\right) \\
\hat{\mathbf{s}}_{N_{t}-1}=\text { décision }\left((\tilde{\mathbf{y}})_{N_{t}-1}-\hat{\mathbf{s}}_{N_{t}} \mathbf{L}_{N_{t}-1, N_{t}}\right) \\
\vdots \\
\hat{\mathbf{s}}_{1}=\text { décision }\left((\tilde{\mathbf{y}})_{1}-\hat{\mathbf{s}}_{N_{t}} \mathbf{L}_{1, N_{t}}-\ldots-\hat{\mathbf{s}}_{2} \mathbf{L}_{1,2}\right)
\end{array}\right.
$$

Le décodage SIC présenté ici utilise le critère ZF mais il est également possible d'utiliser le critère MMSE. Au lieu de décoder dans l'ordre naturel, on peut décoder les symboles du plus puissant au moins puissant à partir de la matrice $\mathbf{H}$. L'algorithme de décodage par soustraction successive des interférences avec ordonnancement (OSIC) donne de meilleures performances avec une complexité sensiblement supérieure.

\section{CodAGE SPATIO-TEMPOREL EN BLOC}

\section{A. Introduction}

Les codes spatio-temporel se divisent en deux familles: les codes spatio-temporel en treillis (STT) et les codes spatiotemporel en bloc (STB). Les codes STT [16] sont une généralisation des modulations codées en treillis aux canaux MIMO. Bien que les performances obtenues par ces codes soient excellentes, la complexité du décodage est exponentielle avec le rendement. En effet, pour le décodage, on utilise une version vectorisée du décodeur de Viterbi. Dans ce chapitre, nous ne présenterons que les codes spatio-temporel en bloc qui sont plus intéressants sur le plan pratique.

\section{B. Codes spatio-temporels en bloc orthogonaux}

Pour le cas $N_{t}=2$ et $N_{r}=1$, Alamouti [1] a proposé un code spatio-temporel avec $Q=T=2$ et donc $R_{M I M O}=1$.

A l'instant 1 , les symboles $s_{1}$ et $s_{2}$ sont transmis respectivement sur les antennes 1 et 2 puis à l'instant 2 , les symboles $-s_{2}^{*}$ et $s_{1}^{*}$ sont transmis sur les antennes 1 et 2 . Ainsi sous forme matricielle, on a :

$$
\mathbf{C}_{S T B C, 2}=\left[\begin{array}{cc}
s_{1} & -s_{2}^{*} \\
s_{2} & s_{1}^{*}
\end{array}\right]
$$

Le code présente la propriété d'être orthogonal car on a

$$
\mathbf{C}_{S T B C, 2} \mathbf{C}_{S T B C, 2}^{H}=\left(\left|s_{1}\right|^{2}+\left|s_{2}\right|^{2}\right) \mathbf{I}_{2}
$$

Ce système peut se mettre sous la forme équivalente

$\mathcal{Y}=\left[\begin{array}{l}y_{11} \\ y_{12}^{*}\end{array}\right]=\left[\begin{array}{cc}h_{11} & h_{12} \\ -h_{12}^{*} & h_{11}^{*}\end{array}\right]\left[\begin{array}{l}s_{1} \\ s_{2}\end{array}\right]+\left[\begin{array}{l}n_{11} \\ n_{12}^{*}\end{array}\right]=\mathcal{H} \mathbf{s}+\mathcal{N}$

Pour ce code, le gain de diversité est égal à $\left|h_{11}\right|^{2}+\left|h_{21}\right|^{2}$.

Comme $\mathcal{H}$ est une matrice orthogonale, le décodage au sens du maximum de vraisemblance (MV) s'obtient simplement en multipliant le vecteur reçu par $\mathcal{H}^{H}$,

$$
\tilde{\mathbf{s}}=\mathcal{H}^{H} \mathcal{Y}=\left(\left|h_{11}\right|^{2}+\left|h_{12}\right|^{2}\right) \mathbf{s}+\tilde{\mathbf{N}}
$$

Puisque le vecteur de bruit $\tilde{\mathbf{N}}=\mathcal{H}^{H} \mathcal{N}$ est encore gaussien de moyenne nulle et de covariance $\rho N_{0} \mathbf{I}_{2}$, on peut alors décoder les deux symboles séparément.

Par rapport à une transmission SISO, le rapport signal à bruit pour chaque symbole est égal à $\left(\left|h_{11}\right|^{2}+\left|h_{12}\right|^{2}\right) E_{s} / N_{0}$.

L'utilisation du code d'Alamouti $N_{t}=2$ et $N_{r}=1$ permet en ajoutant un code correcteur d'erreur puissant de s'approcher de la capacité du canal MIMO. Si nous ajoutons une seconde antenne à la réception $\left(N_{r}=2\right)$, le code d'Alamouti n'est plus optimal.

Le code d'Alamouti est le seul code orthogonal complexe permettant d'atteindre la diversité maximale avec un rendement 
égal à $R_{M I M O}=1$. Il existe seulement quelques autres codes orthogonaux complexes ayant un rendement inférieur à 1 [15]. Par exemple pour $N_{t}=3, N_{r}=1, Q=3$ et $T=4$ et donc $R_{M I M O}=3 / 4$ on a le matrice code suivante:

$$
\mathbf{C}_{S T B C, 3}=\left[\begin{array}{cccc}
s_{1} & s_{2} & s_{3} & 0 \\
-s_{2}^{*} & s_{1}{ }^{*} & 0 & -s_{3} \\
-s_{3}^{*} & 0 & s_{1}{ }^{*} & s_{2}
\end{array}\right]
$$

Comme précédemment, la structure orthogonale permet de décoder simplement ce code.

\section{Codes spatio-temporels en bloc presque orthogonaux}

Sous réserve de sacrifier la propriété d'orthogonalité, il est possible de construire des codes de rendement supérieur ou égal à 1 . Par exemple des structures ont été proposées pour $N_{t}=4$ [9] avec un rendement de 1. La matrice code est la suivante :

$$
\mathbf{C}_{S T B C, 4}=\left[\begin{array}{cccc}
s_{1} & -s_{2}^{*} & -s_{3}^{*} & s_{4} \\
s_{2} & s_{1}{ }^{*} & -s_{4}^{*} & -s_{3} \\
s_{3} & -s_{4}^{*} & s_{1}{ }^{*} & -s_{2} \\
s_{4} & s_{3}^{*} & s_{2}{ }^{*} & s_{1}
\end{array}\right]
$$

Cette matrice est obtenue à partir de deux matrices d'Alamouti et d'une transformée de Hadamard.

Par rapport aux codes STBC orthogonaux, la multiplication du vecteur reçu par la matrice $\mathcal{H}^{H}$ ne permet pas d'obtenir les performances du décodeur MV. En effet, contrairement aux codes STBC orthogonaux on a :

$$
\mathcal{H}^{H} \mathcal{H}=\sum_{i=1}^{4}\left(\left|h_{1 i}\right|^{2}\right) \mathbf{I}_{4}+\mathbf{J}
$$

où la matrice $\mathbf{J}$ est la matrice d'interférence qui contient plusieurs éléments non nuls car le code n'est pas orthogonal.

On peut alors effectuer un décodage linéaire (ZF ou MMSE) ou utiliser un décodeur par sphère [19] pour déterminer la solution au sens du MV avec une complexité limitée. En effet, dans ce contexte, un décodeur MV cherche la solution suivante :

$$
\hat{\mathbf{c}}_{\mathrm{ml}}=\arg \min _{\mathbf{c} \in \Lambda_{L}}\|\mathbf{y}-\mathbf{H c}\|^{2}
$$

Ce problème est équivalent à rechercher le point le plus proche du point reçu dans un réseau de point défini par la matrice du canal MIMO H. L'utilisation d'un décodeur par sphère limite la recherche de la solution MV dans une hypersphère dont le rayon est choisi afin de garantir qu'elle comprenne au moins une solution. Le choix du rayon est fonction de la variance du bruit.

Sur la figure 6, nous comparons les performances $T E B=$ $f\left(E_{B} / N_{0}\right)$ de différentes structures. Nous pouvons vérifier que le décodeur MV donne des performances sensiblement meilleures que le décodeur ZF. Pour un TEB $=10^{-4}$, le code STB presque orthogonal $(3,1)$ donne $2 d B$ de gain de diversité par rapport au code d'Alamouti $(2,1)$. Il donne pratiquement les mêmes performances que le code STB presque orthogonal avec $(4,1)$. Cette figure illustre également l'intérêt d'utiliser si possible un système MIMO $(2,2)$ plutôt que $(4,1)$ !

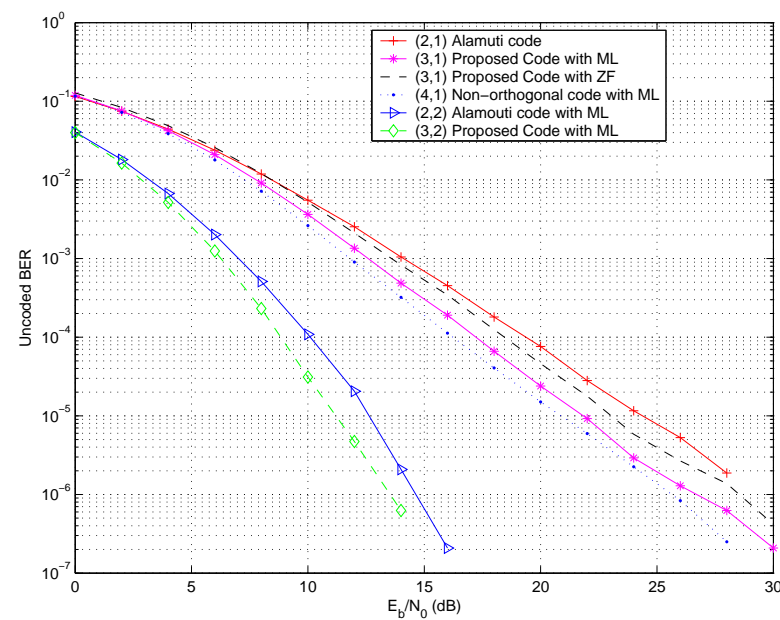

Fig. 5. $T E B=f\left(E_{B} / N_{0}\right.$ de différentes structures pour des canaux de Rayleigh plats

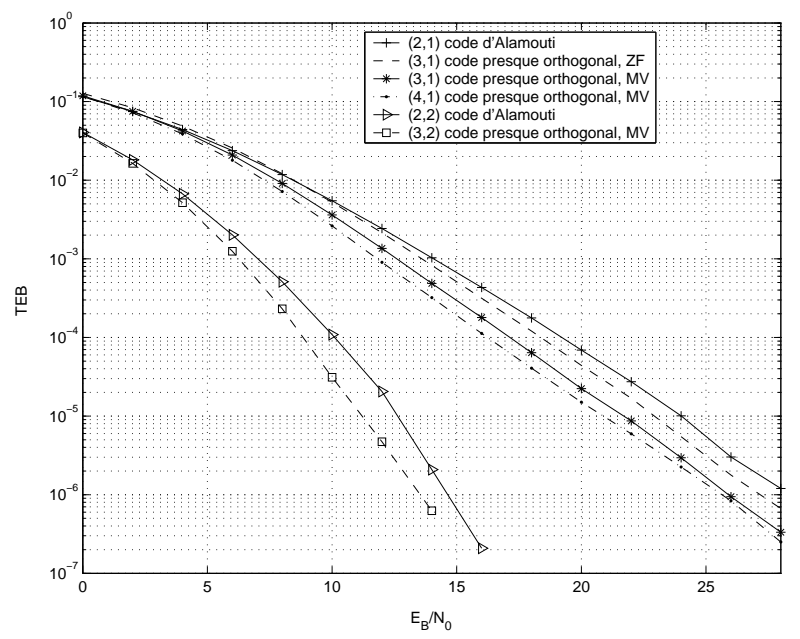

Fig. 6. $T E B=f\left(E_{B} / N_{0}\right.$ de différentes structures pour des canaux de Rayleigh plats

\section{D. codes DAST et TAST}

les codes spatio-temporels DAST (Diagonal Algebraic Space Time Block) et TAST (Threaded Algebraic Space Time Block) sont une généralisation des modulations tournées introduites pour le canal de Rayleigh par Boullé et Belfiore.

Le codage spatio-temporel DAST [4] est un code de rendement $R_{M I M O}=1$ avec $Q=T=N_{t}$ construit à partir d'une matrice de rotation $\mathbf{M}$. La matrice code est de la forme

$$
\mathbf{C}=\operatorname{diag}\left(t_{1}, t_{2}, \ldots, t_{N_{t}}\right)
$$

avec $\mathbf{t}=\left[\begin{array}{llll}t_{1} & t_{2} & \ldots & t_{N_{t}}\end{array}\right]^{T}=\mathbf{M s}$

La matrice de rotation $\mathbf{M}$ est le produit de la matrice de Fourier $\mathbf{F}_{N_{t}}$ de dimension $N_{t} \times N_{t}$ et de la matrice diagonale composée des puissances successives du paramètre de rotation $\alpha$ :

$$
\mathbf{M}=\mathbf{F}_{N_{t}} \operatorname{diag}\left[1, \alpha, \alpha^{2}, \ldots, \alpha^{N_{t}-1}\right]
$$

Les codes DAST atteignent la diversité maximale de $N_{t} N_{r}$ grâce à l'extension de constellation. Nous choisirons le paramètre $\alpha$ afin de maximiser le gain de codage. 
Cette maximisation est effectuée par recherche exhaustive ou en utilisant les propriétés de la théorie des nombres. Par exemple, pour $N_{t}=2$ et une modulation MDP4 des symboles $s_{i}$, on obtient $\alpha=\exp \left(\frac{j \pi}{4}\right)$

$$
\mathbf{C}=\left(\begin{array}{cc}
s_{1}+s_{2} \exp j \frac{\pi}{4} & 0 \\
0 & s_{1}+s_{2} \exp j \frac{3 \pi}{4}
\end{array}\right)
$$

Les codes TAST (threaded algebraic space time)[5] sont une généralisation des codes DAST . Ces codes permettent d'atteindre le compromis optimal entre gain de diversité et de multiplexage. Pour $N_{t}=2, N_{r} \geq 2$ et un rendement $R_{M I M O}=2$, on a la matrice de code suivante :

$$
\mathbf{C}=\left(\begin{array}{cc}
s_{1}-\psi_{t t} s_{2} & \psi_{t t}^{1 / 2}\left(s_{3}+\psi_{t t} s_{4}\right) \\
\psi_{t t}^{1 / 2}\left(s_{3}-\psi_{t t} s_{4}\right) & s_{1}+\psi_{t t} s_{2}
\end{array}\right)
$$

où $\psi_{t t}=\mathrm{e}^{j \zeta_{t t}}$ et $\zeta_{t t}$ est un paramètre réel à optimiser pour obtenir le meilleur gain de codage. On a $\zeta_{t t}=0.5$ pour une modulation MDP4 et $\zeta_{t t}=0.448$ pour une modulation MAQ16.

Comme pour les codes non orthogonaux, on peut utiliser un décodage linéaire (ZF ou MMSE), non linéaire (SIC) ou par sphère.

Sur la figure 7 , nous comparons les performances taux d'erreurs mots $T E M=f\left(E_{B} / N_{0}\right)$ du code d'Alamouti $(2,2)$ avec le code TAST $(2,2)$ pour un débit binaire de 4 bits par intervalle de temps élémentaire ( MAQ 16 pour le code d'Alamouti et MAQ 4 pour le code TAST). On peut voir que le code TAST $(2,2)$ avec décodage MV donne un gain d'environ $2 \mathrm{~dB}$ par rapport au code d'Alamouti $(2,2)$.

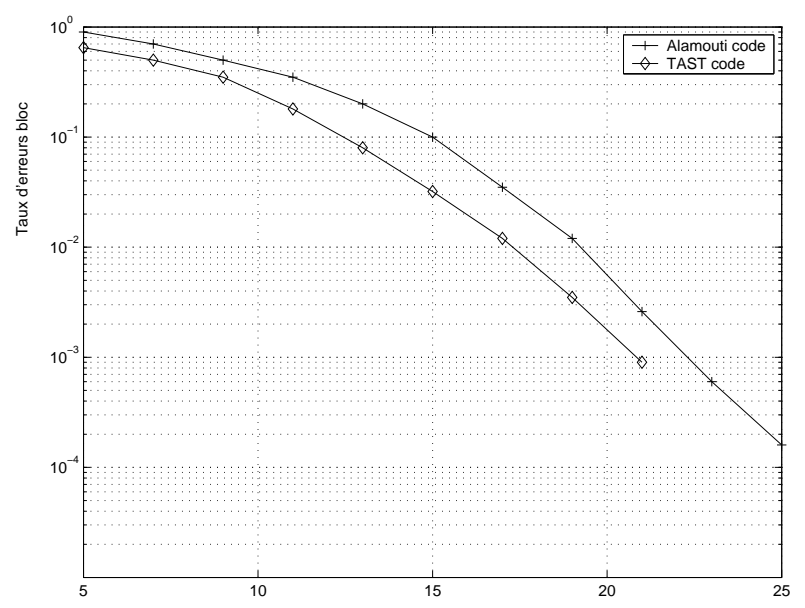

Fig. 7. $T E M=f\left(E_{B} / N_{0}\right)$ du code d'Alamouti vs code TAST pour $N_{t}=$ $N_{r}=2$

\section{CODAGE POUR LES SYSTÈMES MIMO}

Afin de s'approcher de la capacité des canaux MIMO, il est nécessaire ajouter un code correcteur d'erreurs comme un code convolutif ou un turbo code au code spatio-temporel. Le décodeur MIMO à sorties binaires doit alors être remplacé par un décodeur à sorties pondérées. Comme dans la turbo égalisation, sous réserve d'avoir ajouté un entrelaceur entre le code correcteur d'erreurs et le code spatio-temporel, l'information en sortie du décodeur de canal peut être renvoyée au décodeur MIMO comme information a priori. Lors de la seconde itération, le décodeur MIMO utilisera alors conjointement l'information a priori et l'information issue du canal de transmission. La figure 8 présente le synoptique d'une telle chaine de transmission.

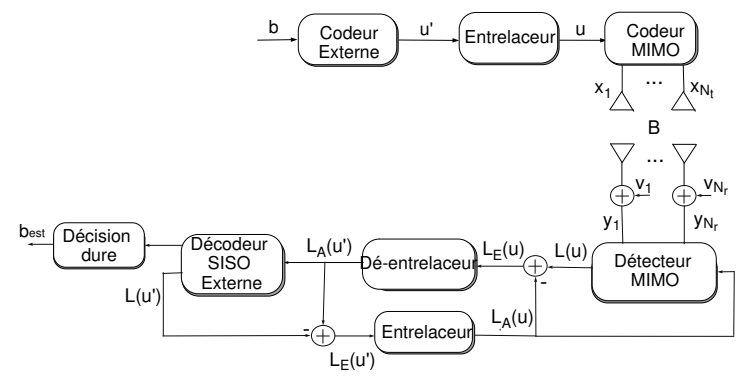

Fig. 8. système MIMO avec codage de canal

Un décodeur MIMO à entrées et sorties pondérées doit calculer les logarithmes de rapport de vraisemblance $L\left(u_{p m} \mid \mathbf{y}\right)$ pour tous les bits transmis :

$$
L\left(u_{p m} \mid \mathbf{y}\right)=\ln \frac{\mathrm{P}\left(u_{p m}=+1 \mid \mathbf{y}\right)}{\mathrm{P}\left(u_{p m}=-1 \mid \mathbf{y}\right)} .
$$

Ce calcul implique une marginalisation sur l'ensemble des $2^{N_{t} M_{c}}$ vecteurs $\mathbf{u}$ :

$L\left(u_{p m} \mid \mathbf{y}\right)=\ln \frac{\mathrm{P}\left(u_{p m}=+1\right)}{\mathrm{P}\left(u_{p m}=-1\right)}+\ln \frac{\sum_{\mathbf{u} \in \mathbb{U}_{p m,+1}} \mathrm{p}(\mathbf{y} \mid \mathbf{u}) \mathrm{P}\left(\mathbf{u} \mid u_{p m}\right)}{\sum_{\mathbf{u} \in \mathbb{U}_{p m,-1}} \mathrm{p}(\mathbf{y} \mid \mathbf{u}) \mathrm{P}\left(\mathbf{u} \mid u_{p m}\right)}$.

$M_{c}$ est le nombre de bit par symbole complexe et $\mathbb{U}_{p m,+1}$ est l'ensemble des $2^{N_{t} M_{c}-1}$ vecteurs $\mathbf{u}$ avec $u_{p m}=+1$.

Comme pour le décodeur par sphère, en pratique, il est possible de réduire la complexité de ce calcul en ne considérant que les vecteurs $\mathbf{u}$ pour lesquels la distance $\|\mathbf{y}-\mathbf{H c}\|$ est petite.

Une autre solution pour calculer les logarithmes de rapport de vraisemblance $L\left(u_{p m} \mid \mathbf{y}\right)$ consiste à utiliser un récepteur linéaire MMSE modifié pour prendre en compte l'information a priori [20]. Ce détecteur à entrées et sorties pondérées présente un bon compromis performance complexité.

La figure 9 présente les courbes de performance $T E B=$ $f\left(E_{B} / N_{0}\right)$ pour un système MIMO V-BLAST $(4,4)$. On considère que les canaux de Rayleigh sont indépendants et que le récepteur connaît parfaitement ces canaux. La longueur des trames de bits d'information est égale à 9216 bits et le codeur de canal choisi est un code convolutif de rendement $(7,5)$. La modulation choisie est la modulation MDP4; on a donc une efficacité spectrale de $4 \mathrm{bit} / \mathrm{s} / \mathrm{Hz}$. Les performances du décodeur par sphère à liste (TMD) en limitant le nombre maximum de vecteurs considérés à 128 ( en moyenne on a environ 40 vecteurs considérés) sont comparées avec celles du décodeur MMSE pour 4 itérations. Il est intéressant de noter que le décodeur par sphère à liste converge plus rapidement que le décodeur MMSE. Cependant, la complexité du décodeur MMSE reste inférieure à celle du décodeur par sphère. 


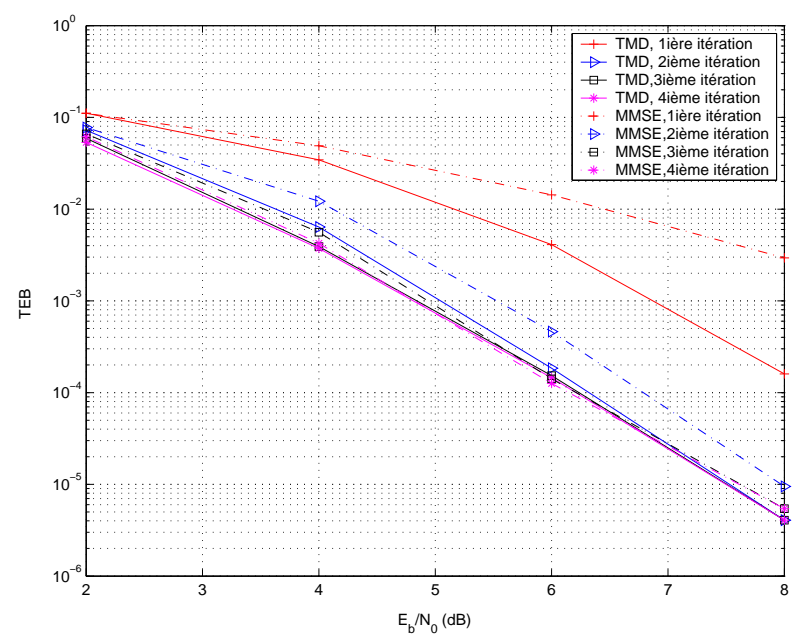

Fig. 9. $T E B=f\left(E_{B} / N_{0}\right)$ pour un système MIMO V-BLAST $4 \times 4$, modulation MPD4, code convolutif rendement $1 / 2$

\section{CODAGE SPATIO-TEMPOREL EN BLOC SUR CANAUX MIMO SÉLECTIFS EN FRÉQUENCE}

Dans les paragraphes précédents nous n'avons considéré que des canaux de transmission non sélectif en fréquence. Cependant, dans la plupart des applications, les canaux de transmission sont sélectifs en fréquence et pour les systèmes MIMO, l'égalisation est rendue plus difficile à cause du nombre de canaux de transmission.

Hormis dans le cas du code d'Alamouti où il est possible d'égaliser dans le domaine temporel en utilisant la technique proposée par Lindskog et Paulraj [13], l'approche classique consiste à associer les codes spatio-temporels avec la technique OFDM (orthogonal frequency division multiplexing).

La technique OFDM permet de convertir un canal sélectif en fréquence en un ensemble de sous-canaux non sélectifs en fréquence. L'utilisation de l'intervalle de garde permet de supprimer totalement l'interférence intersymbole.

Suivant les propriétés des canaux de transmission, le codage STB peut être effectué dans le domaine temporel (STBCOFDM) [11] ou dans le domaine fréquentiel (SFBC-OFDM) [3].

\section{A. Estimation des canaux de transmission}

Par rapport aux canaux SISO, l'estimation des canaux MIMO sélectifs en fréquence est plus difficile car il y a plus de paramètres à estimer et moins de puissance par émetteur [10]. L'estimation de canal nécessite en général des symboles pilotes qui réduisent la puissance utile transmise et l'efficacité spectrale. Comme les systèmes MIMO ont plus de paramètres à estimer, le nombre de symboles pilotes peut devenir important.

Pour les systèmes MIMO-OFDM, l'approche la plus commune consiste à utiliser les symboles pilotes pour estimer le canal au sens du critère des moindres carrés puis à utiliser une technique d'interpolation [12] et [18]. En plus de la répartition des pilotes sur l'axe du temps et de la fréquence qui dépend des propriétés du canal de transmission, dans les systèmes MIMO, la séquence d'apprentissage doit aussi être optimisée (orthogonalité, ...)[2]. Pour ces systèmes, il est aussi possible d'utiliser des algorithmes adaptatifs [14]. Dans la plupart des architectures, les données ne sont pas utilisées pour améliorer l'estimation des canaux de transmission. Cependant comme en pratique il est toujours difficile d'estimer les canaux de transmission, une approche conjointe semble un axe prometteur pour améliorer la robustesse des systèmes et éventuellement réduire le nombre de pilotes nécessaire à la transmission. De nombreux travaux sur l'estimation aveugle ou semi aveugle sont actuellement en cours.

\section{APPLICATIONS DES SYSTÈMES MIMO ET PERSPECTIVES}

Les systèmes MIMO commencent à être suffisamment matures pour être envisagés dans les futurs standards de télécommunications. Dans beaucoup d'applications, les systèmes MIMO sont associés aux techniques multiporteuses.

Pour les futurs réseaux LAN, le standard IEEE802.11n qui doit être achevé en 2006 prévoit d'utiliser les systèmes MIMO pour augmenter les débits utiles. L'objectif est de transmettre des débits de $100 \mathrm{Mbit} / \mathrm{s}$ à une distance de 100m [22]. Bien que le standard soit en cours d'élaboration, des sociétés comme Airgo, Athéros ou Comsis en France proposent déjà des circuits électroniques ou des blocs de propriété intellectuelle atteignant ces objectifs et qui permettent de réaliser une chaîne de transmission avec des codes STB -OFDM.

Pour les réseaux d'accès sans fil large bande [23], le standard IEEE 802.20 en cours de développement est également basé sur les systèmes MIMO-OFDM pour fournir des débits maximum de $1 \mathrm{Mbit} / \mathrm{s}$ par utilisateur et supporter une mobilité allant jusqu'à $250 \mathrm{Km} / \mathrm{h}$. Cependant, les travaux sur les systèmes MIMO dans un contexte multi-utilisateur et multi cellulaire restent assez limités. Ces aspects doivent être traités avant de pouvoir envisager un déploiement dans des systèmes de réseaux cellulaires.

Pour le mode HSDPA (High Speed downlink packet access) de la 3ième génération de radiotéléphones, seul le code d'Alamouti est envisagé pour permettre une diversité spatiale à l'émission. La taille et le cout du terminal limitent pour l'instant le déploiement des systèmes MIMO. Il est aussi certain que les travaux sur les systèmes MIMO dans un contexte multiutilisateur et multi cellulaire restent assez limités. Ces aspects sont en cours de développement dans les différents laboratoires mondiaux.

\section{CONCLUSION}

Dans cet article, nous avons présenté les principes des systèmes MIMO pour les systèmes de communication sans fil. Nous avons montré que la théorie de l'information permet de déterminer les limites théoriques de ces systèmes. Ensuite, nous avons présenté les différentes familles de codes spatiotemporels et les algorithmes de décodage associés. Finalement, nous avons montré qu'en concaténant le code STB avec un code correcteur d'erreur, il est possible de s'approcher de la capacité du canal MIMO. Il reste cependant encore beaucoup de points à approfondir comme l'étude et la modélisation des canaux de propagation MIMO, la réduction de complexité de ces architectures (RF et traitement du signal) et la conception d'antennes de petites tailles. 


\section{REFERENCES}

[1] Alamouti, S. M. "A simple transmit diversity technique for wireless communications", IEEE Journal on Selected Areas on Communication, 16, 1451-1458, 1998.

[2] Barhumi, L., Leus, G., \& Moonen, M, "Optimal training design for MIMO-OFDM systems in mobile wireless channels", IEEE Transaction on Signal Processing, 51, 1615-1624, 2003.

[3] H. Bolcskei and A. J. Paulraj, "Space-frequency coded broadband OFDM systems", Proceedings of IEEE WCNC, vol.1, pp.1-6, 2000.

[4] M. O. Damen, K. Abed-Meraim, J. C. Belfiore, "Diagonal Algebraic Space Time Block Codes", IEEE Trans. on Information Theory, vol. IT48,n³, pp. 628-636, March 2002.

[5] H. El Gamal, M. O. Damen, "Universal Space Time Coding", IEEE Trans. on Information Theory, vol. IT-49, pp. 1097-1119, May. 2003.

[6] Foschini, G. J., \& Gans, M. J, "On the limits of wireless communications in fading environment when using multiple antennas", Wireless Personal Communications, 6, 311-335, 1998.

[7] Foschini, G. J., Golden, G. D., Valenzuela, R. A., \& Wolniansky, "Simplified processing for high spectral efficiency wireless communication employing multi-element arrays", IEEE Journal on Selected Areas on Communications, 17, 1841-1852, 1999.

[8] B. Hochwald, S. Ten Brink, "Achieving near capacity on a multiple antenna channel", IEEE Trans. on Comm., vol. COM-51,pp. 389-399, march 2003.

[9] Jafarkhani, H, "A quasi-orthogonal space-time block code", IEEE Transaction on Communication, 49, 1-4, 2000.

[10] C. Komninakis and C. Fragouli and A. H. Sayed and R.D Wesel, "Multi-input Multi-output Fading channel tracking and equalization using Kalman estimation", IEEE Journal on Selected Areas on Communications, vol.20, pp.1211-1226, 2002

[11] K.F. Lee and D.B. Williams, "A space-time coded transmitter diversity technique for frequency selective fading channels", Proceedings of Sensor Array and Multichannel Signal Processing Workshop,pp.149-152, 2000.

[12] S. Liang and W. Wu, "Channel estimation based on pilot subcarrier in space-time block coded OFDM system", Proceedings of IEEE ICCT, vol.2,pp.1795-1798, 2003.

[13] E. Lindskog and A. Paulraj, "A transmit diversity scheme for channels with intersymbol interference", Proceedings of IEEE International Conference on Communication (ICC), pp.307-311, 2000.

[14] T. Roman and M. Enescu and V. Koivunen,"Time domain method for tracking dispersive channels in MIMO-OFDM systems",Proceedings of IEEE ICASSP, vol.4,pp.393-396, 2003

[15] Tarokh, V., Jafarkhani, H., \& Calderbank, A, "Space-time block codes from orthogonal designs", IEEE Transaction on Information Theory, 45, 1456-1467, 1999.

[16] Tarokh, V., Seshadri, N., \& Calderbank, A. R, "Space-time codes for high data rate wireless communication: Performance criterion and code construction", IEEE Transaction on Information Theory, 44, 744-765, 1998.

[17] Telatar, E, "Capacity of multiple antenna Gaussian channels", AT\&T Bell Laboratories, Technical Report 1995.

[18] D. M. Teran and R. P. T. Jimenez, "Channel estimation for STBC-OFDM systems", Proceedings of 5th IEEE SPAWC", pp.688-691, 2004.

[19] E. Viterbo, J. Boutros, "A universal lattice code decoder for fading channels", IEEE Trans. Inform. Theory, vol. IT-45,pp. 1639-1642, July 1999.

[20] X. Wang, V. Poor, "Iterative (turbo) soft interference cancellation and decoding for coded CDMA", IEEE Trans. on Communications, vol. COM47,pp. 1046-1060, July 1999.

[21] Y. Xin, Z. Wang, G. B. Giannakis, "Space time diversity systems based on linear constellation precoding", IEEE Trans. on Wireless communications, vol. $2 \mathrm{n}^{\circ} 2$,pp. 294-309, Mar. 2003.

[22] http://grouper.ieee.org/groups/802/11/Reports.

[23] http://grouper.ieee.org/groups/802/20.

[24] RAN WG1 N:23, R1-02-0142. MIMO system simulation methodology. 